\title{
Belphégor
}

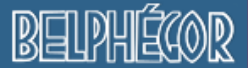

Littérature populaire et culture médiatique

15-1 | 2017

1936: les Jeux olympiques dans la presse

internationale

\section{Thierry Groensteen, La bande dessinée au tournant}

\section{Vittorio Frigerio}

\section{(2) OpenEdition \\ Journals}

Electronic version

URL: http://journals.openedition.org/belphegor/823

DOI: $10.4000 /$ belphegor.823

ISSN: 1499-7185

Publisher

LPCM

\section{Electronic reference}

Vittorio Frigerio, «Thierry Groensteen, La bande dessinée au tournant », Belphégor [Online], 15-1 | 2017, Online since 16 April 2017, connection on 24 September 2020. URL : http://journals.openedition.org/ belphegor/823 ; DOI : https://doi.org/10.4000/belphegor.823

This text was automatically generated on 24 September 2020 .

\section{(c) $($ ) $(9)$}

Belphégor est mis à disposition selon les termes de la Licence Creative Commons Attribution - Pas d'Utilisation Commerciale - Pas de Modification 4.0 International. 


\section{Thierry Groensteen, La bande dessinée au tournant}

\section{Vittorio Frigerio}

\section{REFERENCES}

Groensteen, Thierry. La bande dessinée au tournant. Bruxelles : Les Impressions nouvelles / La Cité international de la bande dessinée et de l'image, 2017. 121 p. ISBN :

978-2-87449-436-9

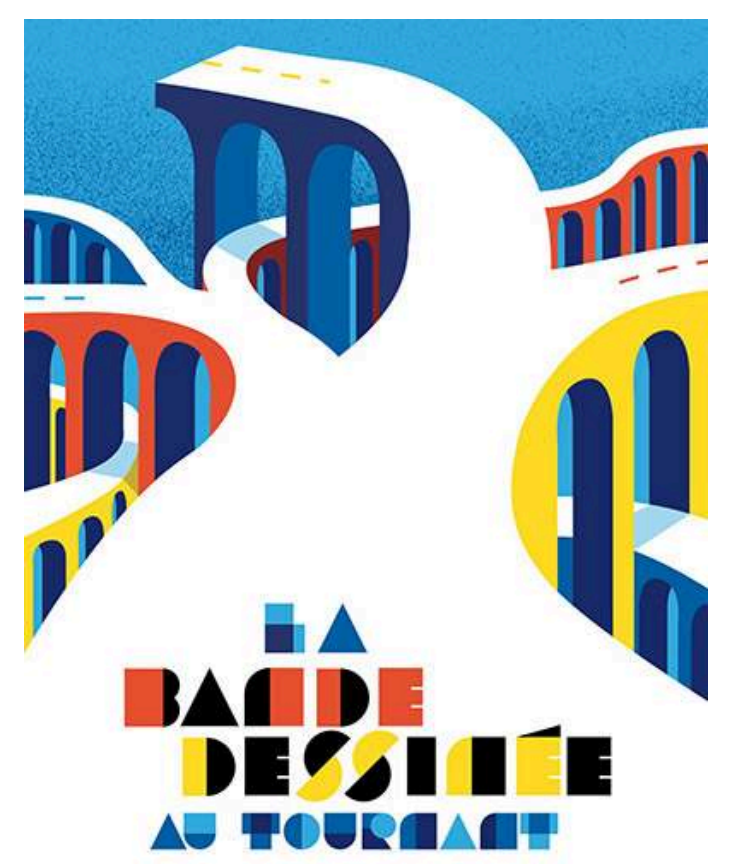

THIERRY
GROENSTEEN 
1 Avec ce petit livre nerveux, Thierry Groensteen vient nous donner un nouveau « bilan décennal » (p. 111) pour faire suite à son livre de 2006, Un objet culturel non identifié. État des lieux de la situation du monde de la bande dessinée, vérification de la plus ou moins grande justesse des prévisions et des jugements d'il y a dix ans (les siens et ceux d'autres critiques), suite de réflexions à bâtons rompus sur cet OCNI (pour emprunter l'acronyme qu'emploie Groensteen pour désigner son « objet culturel non identifié ») dont la présence dans le champ culturel européen est désormais indéniable et apparemment permanente : voilà en quoi consiste, dans ses grandes lignes, cet ouvrage. Bien documenté, farci de suffisamment de chiffres et de statistiques pour étayer avec assurance ses arguments, ce long essai touche à nombre de points importants pour comprendre quelle est l'image que l'on se fait de nos jours de la bande dessinée, quels sont ses producteurs, ses éditeurs, ses lecteurs, et surtout pour tenter de parvenir à quelques conclusions sur la question éternelle de la "légitimation» de la bande dessinée dans le cadre des productions culturelles contemporaines.

2 En fait, malgré quelques jugements occasionnels méprisants et vaguement anachroniques comme ceux d'Alain Finkielkraut - auxquels, selon l'auteur, on accorde une importance disproportionnée qui trahit un fond d'insécurité chez les praticiens du domaine - il semblerait de prime abord que la longue bataille pour l'obtention d'une légitimité culturelle soit maintenant définitivement gagnée. Les grandes maisons d'édition qui encore naguère n'auraient jamais voulu avoir quoi que ce soit à faire avec un produit considéré, au mieux, paralittéraire, mettent des « romans graphiques » dans leur catalogue. Une politique de reconnaissance et de mise en valeur du patrimoine se généralise, avec la publication d'éditions soignées des grandes séries historiques, pourvues d'un certain appareil critique. Les champs dans lesquels la bande dessinée exerce ses talents se diversifient de plus en plus. À l'autobiographie, domaine pionnier, viennent s'ajouter le documentaire, le reportage, la vulgarisation sous toutes ses formes, et même d'authentiques travaux universitaires. C'est à croire que plus aucun domaine appartenant à la non-fiction n'est désormais à l'abri des incursions de la bande dessinée, outil décidément propre à donner forme à bien des types de réflexion que l'on estimait habituellement éloignés d'elle ou incompatibles. Autre développement positif, le nombre de femmes auteures augmente rapidement, ainsi d'ailleurs que le lectorat féminin, et si le champ reste pour l'instant encore très majoritairement masculin, tout indique que la situation ne cessera de tendre vers un plus grand équilibre. Enfin, la muséification de la bande dessinée semble désormais irréversible. Les expositions dédiées aux grands noms du domaine se sont multipliées au point de devenir monnaie courante, et on peut estimer que la présence de la bande dessinée au sein du paysage muséal français n'est maintenant plus susceptible d'être remise en question.

3 Que de bonnes nouvelles, dira-t-on? Groensteen introduit cependant un nombre de bémols assez important dans son portrait de la situation actuelle du champ. C'est que la bande dessinée n'en reste pas moins tout d'abord, et quoi qu'on en dise, dépendante du commerce, que le "mercantilisme ambient» (26) ne donne pas signe de vouloir s'estomper et que si, symboliquement parlant, c'est gratifiant de voir de grandes maisons d'édition traditionnelles occuper le terrain, cette reconnaissance est aussi simplement dictée par la logique de l'expansion commerciale. Si la diversification des sujets traités en bandes dessinées ne cesse d'augmenter, il existe également une tendance à utiliser celle-ci à des fins de vulgarisation - du genre des manuels « pour les 
nuls » (35) - qui gomme quelque peu les différences entre cette production nouvelle et les vieilles «Belles Histoires de l'Oncle Paul », l'académisme et l'hagiographie régnant toujours en maîtres. Bon nombre d'obstacles demeurent donc toujours et exigeraient d'être surmontés. Pour certains, comme la contradiction permanente entre l'ambition artistique des auteurs et les projets des entreprises, il ne s'annonce pas de solution immédiate. La question de la surproduction actuelle, qui donne un faux air de bonne santé au marché, mais est en réalité « la cause principale de la crise que subit à présent la profession» (104), va aussi être difficile à régler. Le marché des originaux, qui contribue d'un côté à confirmer dans l'esprit du public la valeur artistique de la création de bandes dessinées, provoque également «un affairisme spéculateur débridé » (107) qui affecte la profession en son entier. Les revenus des auteurs demeurent largement insuffisants et leur emploi, dans l'ensemble, extrêmement précaire. Et enfin, la quête de légitimation de la bande dessinée peut provoquer des excès révélateurs d'un sentiment profond d'insécurité, si ce n'est d'infériorité, par rapport à la littérature consacrée.

4 Si la quatrième de couverture de l'ouvrage annonce au lecteur que la «légitimité culturelle [de la bande dessinée] ne fait plus guère débat", Groensteen constate néanmoins que "dans le milieu littéraire la bande dessinée n'est pas encore unanimement considérée comme appartenant pleinement à l'univers du livre et des 'œuvres de l'esprit'« (93). La respectabilité artistique semble maintenant atteinte, mais la respectabilité littéraire repose sur des bases encore fragiles. Le travail de promotion de la bande dessinée dans l'ordre symbolique demeure donc un work in progress, et s'il n'y a qu'une chose dont on doive se réjouir à cause de cela, c'est que dans dix ans nous aurons vraisemblablement à nouveau droit à un état des lieux de la question complet et bien argumenté, comme l'est ce livre.

\section{AUTHOR}

\section{VITTORIO FRIGERIO}

Dalhousie University 